RHIC-PH-3

VERY HIGH ENERGY PROBES

OF THE

QUARK-GLUON PLASMA

T. Ludlam and F. Paige

Brookhaven National Laboratory

March 1984 


\title{
VERY HIGH ENERGY PROBES OF THE QUARK-GLUON PLASMA
}

\author{
T. Ludlam and F. Paige \\ Brookhaven National Laboratory \\ Upton, New York 11973 \\ L. Madansky \\ Johns Hopkins University \\ Baltimore, Maryland 21218
}

\section{INTRODUCTION}

Among the penetrating probes of nuclear matter the most frequently discussed have been those which involve the detection of photons or leptons with $\mathbb{m}_{\mathrm{T}} \simeq \mathrm{P}_{\mathrm{T}}$ $<3 \mathrm{GeV}$. This is the expected range of emission from a hot, thermalized plasma of quarks and gluons. The suggestion has been made that in very high energy collisions of muclei the properties of high $\mathrm{P}_{\mathrm{T}}$ jets may also reflect the characteristics of the muclear medium through which the parent partons have propagated just after the collision. ${ }^{1}, 2$ In this note we expand on the possible uses of such a probe.

\section{HIGH PT JETS}

Consider a high energy muclear collision in which a pair of partons suffers a hard scattering event of the sort which produces the hadronic jets observed at large transverse momentum in pp and $\bar{p}$ p collisions. One or both of the observed jets may be the hadronization of a quark or gluon which has propagated through a substantial volume of quark-gluon plasma produced in the collision. The average properties of such jets may be measurably different from those of "ordinary" jets. We have in mind two possible effects:

1) The hadronization of ordinary jets proceeds as $\mathrm{q} \bar{q}$ pairs are materialized from the QCD vacuum (the "sea"). It may be that a quark or gluon propagating through the real sea of a deconfined plasma hadronizes with quite different properties which could signify the presence of a plasma and provide clues to its characteristics. The parameters of jets in which these differences might show up include the fragmentation function $f(z)$, the transverse momenta of fragmentation products relative to the jet axis, and the flavor content of the leading particles. Specifically, if chiral symmetry is restored in the plasma we may expect an enhanced fraction of strangeness and heavier flavors among. the fragmentation products of the jet. In addition, one should be sensitive to a possible halo of soft particles accompanying the jet, which may arise from "radiative" effects as the parent parton traverses the plasma. Such a component of soft particles in excess of the minimum bias background has been observed in multi-jet events seen in the $\mathrm{UA}-1$ experiment at the CERN $\overline{\mathrm{p}}$ collider.

2) The correlation within pairs of jets should be examined for evidence of the characteristic energy loss behavior suggested by Bjorken. ${ }^{1}$ High energy quarks and gluons traversing the plasma may suffer energy loss comparable to the initial $\mathrm{P}_{\mathrm{T}}$, giving rise to a transverse momentum imbalance in the jet pair which is not 
seen in pp collisions. In the extreme case a hard scatter occurring near the periphery of the plasma volume results in one jet escaping unattenuated while the other is completely absorbed.

- To do these experiments we must detect jets and, in many instances, resolve individual particles within jets. From experience at the CERN collider, we expect that this will require $P_{\mathrm{T}}$ (jet) $>20 \mathrm{GeV}$, and that these experiments will only be carried out in high energy colliding beam machines. Unlike most of the contemplated probes of a quark-gluon plasma, which involve the detection of soft particles amongst the very high multiplicity background, these measurements allow for very selective calorimetric triggers on local transverse energy deposition. Fig. 1 shows a possible detector arm covering 2 units of rapidity centered at $y=$ 0 , and $90^{\circ}$ of azimuth. This detector arm was a design study for the detection of heavy quark jets at CBA. The detector is based on a well-segmented, high resolution calorimeter, and includes tracking chambers capable of resolving individual tracks within jets. The device was designed to have good efficiency for identifying both electrons (transition radiation detectors) and muons associated with jets. The correlation experiment would require two such arms. We have not examined in detail the performance of such a detector arm in a heavy ion collider, but we believe it would be well suited. Again, this is because the studies we are interested in here allow an effective anti-selection of the low $\mathrm{P}_{\mathrm{T}}$ particles which give the most formidable backgrounds in these events.

To estimate the rate for observing jets we take the cross section for $p-p$ collisions and assume this scales as $A^{2}$. The $p+p \rightarrow$ jet $+x$ cross section is given by ISAJET. ${ }^{5}$ The number of jets produced in a year of running is:

$$
N=L \quad T \quad \sigma \quad A^{2}
$$

where $\sigma$ is the cross section in PP collisions, and we take for the luminosity L and time $T$ :

$$
\begin{aligned}
& \dot{\mathrm{L}}=10^{27} \mathrm{~cm}^{-2} \mathrm{sec}^{-1} \\
& \mathrm{~T}=10^{7} \text { sec/machine year }
\end{aligned}
$$

The number of jets per year for various $P_{T}$ thresholds is shown in Fig. 2 as a function of the machine energy.

For heavy ions, the minimum $P_{T}$ for observable jets is higher than in $p-p$ collisions because of the many soft particles produced. For pp collisions at accelerator energies where jets are observed, the particle density (charged plus neutral) at mid-rapidity is:

$$
\frac{d n}{d y} \simeq 4
$$

The KNO multiplicity distribution gives:

$$
\frac{\left(\left\langle n^{2}\right\rangle-\langle n\rangle^{2}\right)^{1 / 2}}{\langle n\rangle} \simeq \frac{1}{2}
$$


With $\left\langle E_{\mathrm{T}}\right\rangle \simeq .4 \mathrm{GeV} /$ particle, we obtain:

$$
\frac{\mathrm{dE}_{\mathrm{T}}}{\mathrm{dy}}=(1.6 \pm 0.8) \mathrm{GeV} \text {, }
$$

where the fluctuation comes just from the multiplicity distribution.

Now consider A + A collisions. Treating these as A independent pp collisions we obtain:

$$
\frac{\mathrm{dE}_{\mathrm{T}}}{\mathrm{dy}}=(1.6 \mathrm{AA} \pm .8 \sqrt{\mathrm{A}}) \mathrm{GeV} .
$$

For $\mathrm{A}=200$ :

$$
\frac{\mathrm{dE}_{\mathrm{T}}(\mathrm{A}=200)}{\mathrm{dy}}=(320 \pm 11.5) \mathrm{GeV} .
$$

Since the highest energy heavy ion collider presently under discussion has $\checkmark \mathrm{S} / \mathrm{A} \simeq 100+100 \mathrm{GeV} / \mathrm{amu}$, no jet will ever dominate the total $\mathrm{E}_{\mathrm{T}}$ distribution. In order to be observed, the $P_{T}$ of the jet must be larger than the $E_{T}$ in a cone large enough to contain it fully, say $\Delta y=1, \Delta \phi=45^{\circ}$. Then for $\mathrm{A}=200$ :

$$
P_{T} \text { (jet) }>\frac{d E_{T}}{d y} \quad \Delta y \frac{\Delta \phi}{2 \pi}=40 \mathrm{GeV} / \mathrm{c} .
$$

Alternatively, we might ask that $2 P_{T}$ (jet) be larger than the fluctuations of $E_{T}$ in the central $\Delta y=2$ interval of rapidity. Hence for $A=200$, requiring $>$ 40:

$$
P_{T}(\text { jet })>\frac{1}{2}\left(\frac{d E_{T}}{d y}\right) \Delta y \times 4=45 \mathrm{GeV} / \mathrm{c} .
$$

These results imply that the observation of jets is possible in high energy collisions of heavy nuclei, but that for these experiments there is a premium on reaching the highest possible energy. Referring to Fig. 2, taking the curve for $\mathrm{P}_{\mathrm{T}}>40 \mathrm{GeV} / \mathrm{c}$, we see that jets should be visible in usable numbers for a $100+$ $100 \mathrm{GeV} / \mathrm{amu}$ collider.

\section{DEEP INELASTIC SCATTERING VIA DRELL-YAN PAIRS}

It may be that high $\mathrm{P}_{\mathrm{T}}$ leptons produced in the massive Drell-Yan continuum can be exploited as a probe of the nuclear medium. Such a probe would be of interest if there were reason to expect observable radiative effects associated with the propagation of these leptons through the plasma. 
A fundamental difference may be the effectively short multiple scattering path compared to the coherence length of the radiative effect (see paper on so-called Landau-Pomeranchuk-Migdal effect). 6 Just how this phenomenon scales in a quark-gluon plasma would be an interesting study. If, however, such an effect is not too small, then one might look for photon pairs or jets that accompany the leptons.

This work was supported by the U.S. Department of Energy under contract $\mathrm{DE}-\mathrm{ACO} 2-76 \mathrm{CH} 00016$ and DE-AC02-76ER03274.

\section{REFERENCES}

1. J.D. Bjorken, Fermilab Pub. 82/59/59-THY (1982).

2. L. Mc Lerran, BNL Report (1983).

3. G. Pancheri and C. Rubbia, Proc. III Internationai Conference on Dltra Relativistic Nucleus-Nucleus Collisions, Nucl. Phys. A418, 117c (1984).

4. G. Benenson, et al., Proc. 1983 DPF Workshop on Collider Detectors, LBL-15973 (1983).

5. F. Paige and S. Protopopescu, BNL-29777; ISAJET Version 4.02 is used, with Eichten, Hinchcliffe, Lane, Quiqq (1984) Structure Functions.

6. T. Stanev, Ch. Vankov, R.E. Streitmatter, R.W. Ellsworth, T. Bowen, Phys. Rev. D25, 1291 (1982).

\section{FIGURE CAPTIONS}

Fig. 1. Single arm heavy quark detector at $\theta=90^{\circ}$, covering \pm 1 units of rapidity and $\pm 45^{\circ}$ in azimuth (Ref. 4). The calorimeter is envisioned to be segmented in tower geometry, with $\approx 100$ cells in the hadronic part and $\approx 1000$ cells in the EM portion (the first 20 radiation lengths). TRD refers to transition radiation detectors.

Fig. 2. The rates for two-jet events with the indicated $P_{T}$ thresholds, as a function of collider energy. The calculation assumes a luminosity of $10^{27} \mathrm{~cm}^{-2} \mathrm{sec}^{-1}$ integrated for $10^{7}$ seconds. 

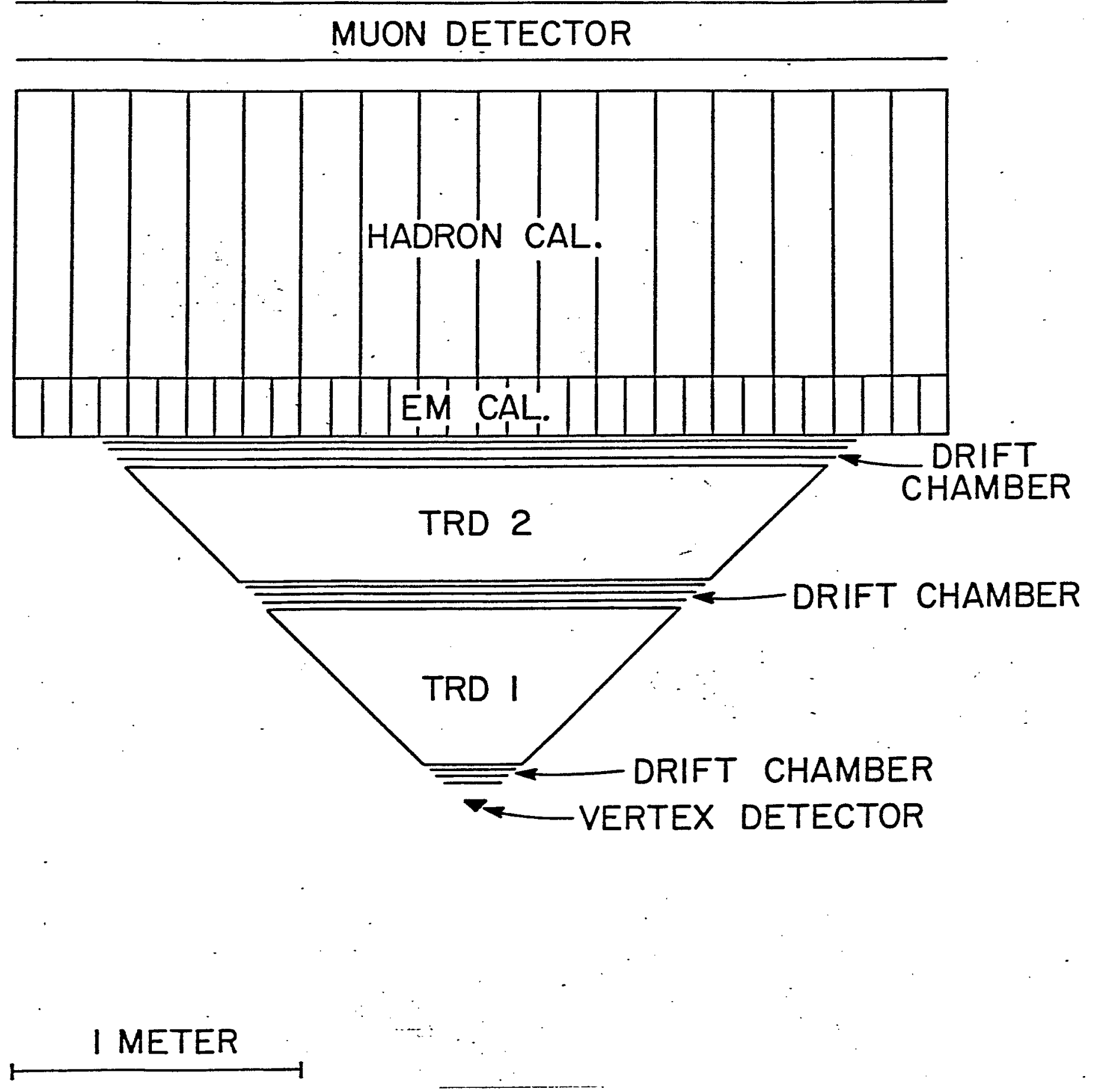

Fig. 1 


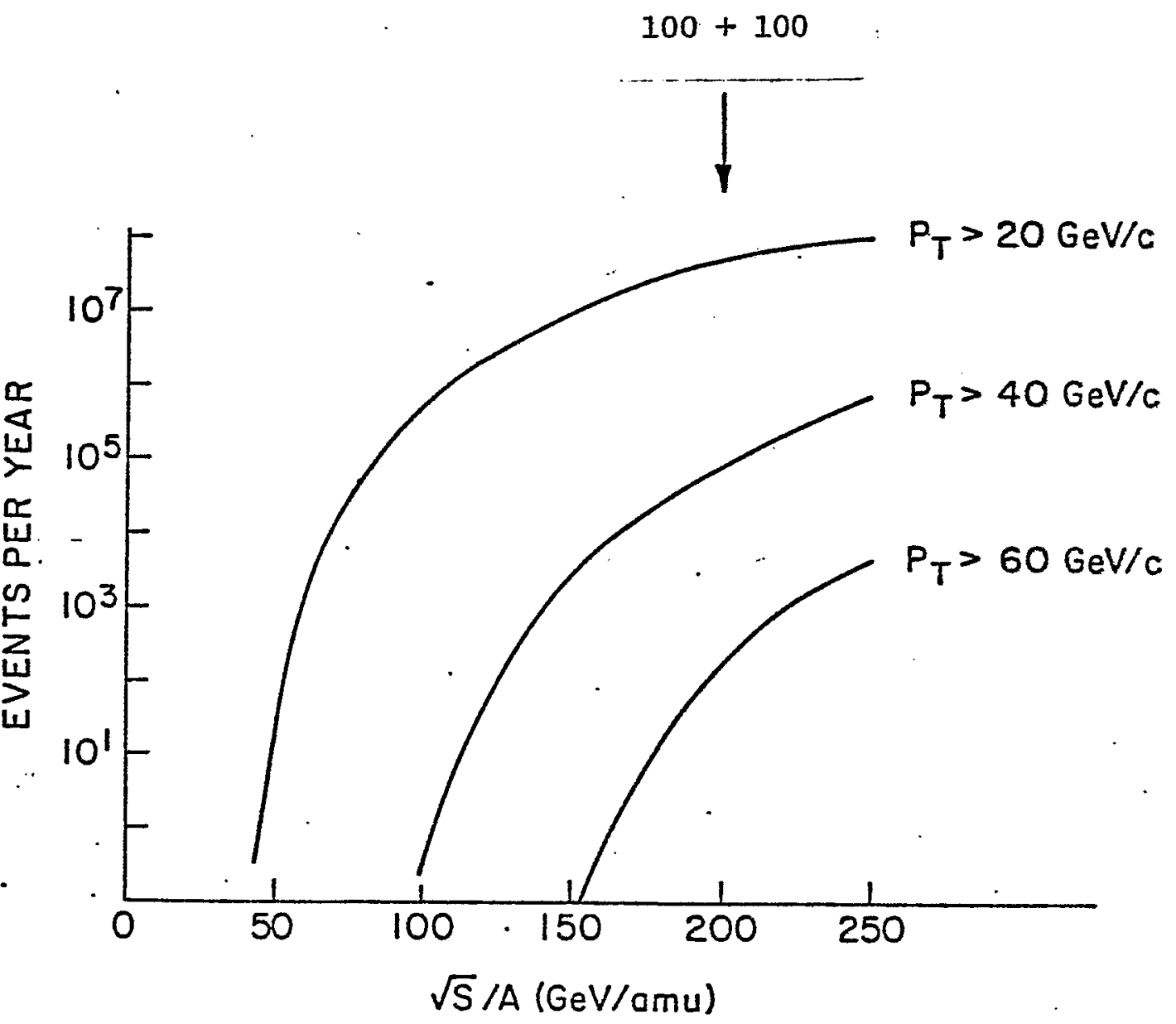

Fig. 2 